\title{
Randomness and nondeterminism: from genes to free will with implications for psychiatry
}

\author{
Ridha Joober, MD, PhD; Sherif Karama, MD, PhD
}

\section{"One Toss of the Dice Will Never Abolish Chance" - Stéphane Mallarmé, 1898}

Randomness and selection are fundamental processes rooted in the very basis of life, as postulated by the theory of evolution. First, mutations are randomly and continuously fed in the gene pool of a population (on average 175 mutations per diploid genome per generation), creating and maintaining over time a high level of genetic diversity. ${ }^{1}$ Second, this genetic diversity is subjected to differential survival and reproduction of individuals facing diverse and often unpredictable environments. This differential fitness is the basic mechanism of natural selection and evolution, a quintessential pillar of modern biology.

In addition to the large number of genetic variations generated by mutations, sexual reproduction (by far the most common mode of reproduction) and recombination are associated with the generation of an enormous amount of genetic variations among individuals. ${ }^{2}$ During parental meiosis, grandparental chromosomes are randomly recombined (on average 1.57 random recombinations for each pair of parental chromosomes, leading to billions of different gametes). ${ }^{2}$ The random assortment of 2 of these gametes ( 1 from each parent) forms the zygote, which will then develop to become a unique individual. The ubiquity of sexual reproduction and recombination, despite the heavy burden associated with this mode of reproduction compared with asexual reproduction (the so-called Sex Paradox), strongly suggests that the processes of generating random variability followed by selection strategies are fundamental to how life starts and thrives. Consequently, all beings conceived via sexual reproduction start life with a toss of genetic dice and are subsequently posted to undergo the trials of life.

Remarkably, these fundamental principles of random generation of variations followed by selection seem to be at odds with how we think and conduct biological research. Indeed, our natural reflexes as scientists, are to study "deterministic mechanisms." Noise and randomness are antithetical to our experimental designs. The dogma in biology stipulates that the genome, once assembled, is the blueprint of development, which is a deterministic process, the cogs of which we try to discover in our laboratories. Of course, this deterministic model allows for environmental contingencies to play a role on how development proceeds, but these are also conceived as deterministic processes.

Notwithstanding the importance of deterministic mechanisms and their role in psychiatric research, ${ }^{3}$ and in the view of the random nature of how beings enter life, as discussed above, should we not expect that the same processes of randomness followed by some kind of selection should be present at all levels of biology and beyond?

In this editorial, we argue that the initial tosses of dice that inaugurate life "will never abolish chance," echoing the title of Mallarmé's famous poem. We will show that randomness is ubiquitous throughout the life cycle and at all levels of analyses. Taking examples from recent developments in molecular biology, neuroscience, psychology and philosophy, we argue that these processes - randomness and selection - are at play at all these levels and may be fundamental to our understanding of complex phenotypes such as psychiatric disorders. Our constant failure to consider these basic aspects of biology and psychology may be at the roots of our chronic struggle to fit mental life in a deterministic framework, however important this framework is.

\section{Randomness and related concepts}

Some concepts discussed here (variation, chance, randomness, chaos, stochasticity, predictability, determinism and nondeterminism) are vast and related topics of discussion in science and philosophy, and are beyond the scope of this editorial. However, the targeted concept in this editorial is randomness in a fundamental sense, like that resulting from dice tosses (result randomness as opposed to process randomness) or attached to quantum observables, and not simply the error of measurements or the residual variances that we are not able to account for because of the incompleteness of our models. The term "nondeterminism" 
is used in the sense of unpredictability that could be related to randomness or other complex dynamics such as that observed in chaotic systems. The term "variation" is used to refer to all sources of variations, including sampling variation and variation due to random or chaotic processes. For more in-depth discussion of these concepts, see Eagle ${ }^{4}$ and Hoefer. ${ }^{5}$

\section{Randomness of cellular molecular processes}

While an important body of work describes the mathematics and biophysics underlying the random processes in cell biology, ${ }^{6}$ it is only in the last 10 years that experimental data about random variation between clonal cells (genetically identical) emerged as a major subject of interest. In a review titled, "What's luck got to do with it? Single cells, multiple fates, and biological nondeterminism," Symmons and Raj discuss the emerging data in the field of single cell biology. ${ }^{7}$ With the tremendous progress in our capacity to measure RNA and protein expression in a single cell, these authors reviewed the data regarding variability between clonal cells as well as the theoretical implications of this variability to our understanding of normal development and pathological conditions. While it is recognized that some of this variability could be explained by "hidden variables" that have not yet been modelled (maybe because they are still not known), there is ample evidence that a significant amount of this variability is related to randomness-generating mechanisms (called diversity generating mapping in this review). Indeed, several studies were specifically designed to disentangle the nondeterministic variability from variability secondary to cellular mechanisms that have not been controlled for. These studies confirmed the presence of intrinsic nondeterministic variability and attributed this variability to the low copy number of a large number of molecules. ${ }^{8,9}$ In another study, Golding and colleagues have been able to count (not just quantify) the number of mRNA molecules in Escherichia coli and model the kinetic of transcription in this organism. ${ }^{10}$ Remarkably, they have shown that transcription happens in quantal bursts and that burst sizes (the number of transcripts) follow a geometrical probability distribution whereas the time intervals between bursts follow an exponential distribution, strongly suggesting that, in addition to central tendencies, random variations are intrinsic to biological mechanisms.

Subsequently, Symmons and Raj asked what could be the role of randomness in basic biological processes. ${ }^{7}$ A first hint indicating that randomness may have a functional role is that many studies reporting on variation of gene transcription among clonal cells found a higher level of variation of transcription factors and other regulatory genes compared with housekeeping genes, ${ }^{11}$ suggesting that selective forces are at play. While much work needs to be done to better understand the role of the probabilistic genotypeto-phenotype mapping in multicellular organisms, there is some evidence that this variation can be implicated in the survival of competing cells during development. For exam- ple, Khare and Shaulsky suggested that the competition among clonal cells might improve the fitness of a multicellular organism by directing survival of fitter cells or genes to the germ line or by eliminating unfit cells. ${ }^{12}$ They also argued that cell-competition mechanisms might have been selectively conserved in multicellular organisms. Some experimental supporting evidence has been reported by Levayer and colleagues, showing that in the imaginal wing disk of Drosophila, the level of variation of the Myc gene determines spatial cell interaction and survival. ${ }^{13}$ More recently, Guillemin and colleagues have shown that randomness in gene expression can be regulated by specific drugs and that an increased variability is associated with higher capacity of cell differentiation, supporting the hypothesis that randomness in gene expression might be an important mechanism underlying cell fate determination. ${ }^{14}$ Thus, it appears that the nondeterministic processes put a limit on our capacity of measuring biological processes and play a significant role in cell fate. ${ }^{15,16}$

An important first implication for psychiatric research is reviewing some aspects of a major paradigm used in genetics of mental disorders, namely twin studies. Indeed, twin studies partition the origin of variation in psychiatric phenotypes into 3 components: additive genetic variation, and common and specific environmental variations. ${ }^{17}$ The specific environmental component of variance, often large in most psychiatric disorders, is the contribution of environmental factors that are not shared between twins as well as residual variance representing measurement errors and/or stochastic variance. However, in case nondeterministic variance plays an important role in development, it is quite possible that a substantial proportion of phenotypic variance attributed to specific environmental factors may be due to nondeterministic processes. ${ }^{17} \mathrm{Re}-$ markably, taking advantage of the peculiar reproductive system in armadillo (a mammal that systematically gives birth to genetically identical quadruplets that are raised under very similar environmental conditions), Ballouz and colleagues showed that a large number of genes are differentially expressed between these genetically identical quadruplets, and that this differential expression is acquired very early during development and remains stable thereafter, giving each armadillo a unique molecular signature individuality. ${ }^{18}$ These differential profiles may be acquired through various epigenetic mechanisms where randomness plays a significant role. As a case in point, they have shown that $X$ chromosome inactivation, a conserved mechanism by which the totality of 1 randomly selected $X$ chromosome in females is silenced, happens at around the embryonic stage of 25 cells in female armadillos. Under such a scenario, each female embryo at the stage of 25 cells acquires a unique distribution of randomly inactivated $X$ chromosomes, following a binomial distribution (equivalent of 25 successive coin tosses) that gives each embryo a unique individuality. They have extended some of their results to gene expression variability in human monozygotic twins and concluded that purely random variations during development could account for 
up to $20 \%$ of the variability between monozygotic twins. These ideas can help to develop new testable hypotheses in relation to mental disorders. There are some data indicating that behavioural phenotypes of females with Turner syndrome (loss of $1 \mathrm{X}$ ) are influenced by the origin of the $\mathrm{X}$ chromosome: females with a maternally inherited $X$ chromosome display more internalized behaviour. ${ }^{19}$ It is possible that in healthy individuals, this trait (and other related traits; e.g., autistic traits) might be determined by the ratio of paternal/maternal inactivated $X$ chromosomes randomly happening in the early stages of development.

Interestingly, some observations indicate that mental disorders are associated with an increase in randomness generating cellular processes. For example, our group reported that patients with schizophrenia have a higher level of de novo mutations than controls. ${ }^{20}$ Furthermore, Igolkina and colleagues compared gene expression between patients with schizophrenia and controls (quantified in cultured neural progenitor cells derived from olfactory neuroepithelium) and concluded that patients with schizophrenia have significantly higher levels of variability of gene expression. ${ }^{21}$ Moreover, a study by Kebir and colleagues has shown that the conversion from a clinical high-risk state to full-fledged psychosis is associated with an increased variability of methylation at several genomic sites. It is therefore possible that the mechanisms generating variability, while important for some basic biological functions, may also lead to psychiatric disorders. ${ }^{22}$

\section{Randommness of the brain connectome and neural function}

Individual variability in brain morphology among twins is well established in the literature, and this variability has also been correlated with behavioural traits. ${ }^{23}$ While most studies stipulate that this individuality may be due to environmental factors, there are now robust studies suggesting that randomness/noise may underly some of this variability in nonvertebrates, and possibly in more evolved animals. In freely moving genetically identical flies, Linnewebber and colleagues. ${ }^{24}$ showed a link between random left/right wiring of the dorsal cluster neurons and behavioural identity, measured by the orientation to visual stimuli. This study was possibly the first to show that behavioural individuality is linked to axonal connectivity arising from random noise during development. Interestingly, Yao and colleagues, combining resting-state functional and structural connectivity analyses, reported that, compared with healthy controls, patients with schizophrenia have significantly higher levels of functional connectivity entropy (an unbiased estimate of unpredictability of connections) in the early stages of illness. ${ }^{25}$

Beyond anatomic determinants of behaviour, there is now ample evidence that randomness plays a major role in neural functioning and behaviour. ${ }^{26}$ In their book The noisy brain: stochastic dynamic as a principle of brain function, Rolls and Deco make a strong case for the fundamental role of stochastic processing of information in the brain, which in turn helps to understand complex human behaviours, including probabilistic decision making, perception, memory recall, attention, short-term memory and possibly creativity. ${ }^{27}$ They also argue that some pathological conditions such as schizophrenia, obsessive-compulsive symptoms and abnormal aging may be understood as hypersensitivity to background noise intrinsic to brain function. ${ }^{28,29}$ The notion of hypersensitivity to background noise, or chaotic dynamics, is another major theme that can be relevant to all levels of analyses discussed in this editorial (molecular, neural, psychological). While chaotic dynamics can be conceived as a deterministic process, its complexity and high level of unpredictability is also compatible with nondeterminism. ${ }^{5}$

\section{Randomness at the psychological level}

In the previous section of this editorial, we discussed the role of randomness at the molecular, cellular and brain structural and functional levels, as well as the possible implications for mental disorders. At a higher level of psychological functioning, theories of decision making and action have been dominated by the causal deterministic model. In this model, the probability of the outcomes of various options are used to estimate expected utilities and engage in a specific decision/action. The dominant framework of probability used in this model is the classic Bayesian model of probability. However, there is now a large body of literature, inaugurated by Tversky and Kahenman, suggesting that human behaviour often violates the classic probability model..$^{30,31}$ In the last 2 decades, the quantum probability model has been proposed in an attempt to explain some characteristics of human behaviour that do not fit with the normative Bayesian model. ${ }^{32,33}$ It is important to note that this emergent quantum cognition field does not stipulate any quantum mechanical nature of the brain as an organ, but proposes the use of the formal quantum probability model (assigning probabilities to observables) as a supplement to the normative model to better understand human decision making and some of the paradoxes posed by the classical model. ${ }^{34,35}$ It is also important to note that, while the quantum probability model seems unfamiliar to most researchers in psychology, it is a model that is formally defined with strong validity in physics. Indeed, there are no epistemic reasons to downplay its pertinence in favour of the classic Bayesian model.

One principle of quantum cognitive probability (among many others) that is not part of the classic probability model is the idea that, under some circumstances (e.g., conflict, ambiguity, confusion, uncertainty) psychological states may exist as a superposition of several potential outcomes. These possibilities exist in a superposition state, and one of them will be observed in a fundamentally random way (we can think about it as the collapse of the wave function in quantum physics). ${ }^{36}$ The classic probability model postulates that at each time, the probability of a decision/action is updated and follows a point-wise, dispersion-free trajectory. However, in the quantum probability model, a decision/action may be represented by an indefinite state, or superposition, 
that collapses to a definite value only at the time of decision/ action (equivalent to observable in quantum physics). Importantly, in the quantum probability model, there is no single trajectory before a decision is made, but rather a "smearing of potentials across states that flows across time." Remarkably, many studies testing various aspects of quantum cognition have recently been published. For example, Kvam and colleagues, in a paper titled, "Temporal oscillations in preference strength provide evidence for an open system model of constructed preference," tracked the dynamic changes in preference strength and showed that the mean preference strength systematically oscillates over time and that the strength of these oscillations is influenced by prior preferences. ${ }^{37}$ They reported that a combination of classic and quantum probability dynamics provides the best model to account for these observations. In another study, Trueblood and colleagues used a unified probability model combining classic Bayesian and quantum probability models to represent human decision making. ${ }^{38}$ They postulated a hierarchy of models of mental representations from fully classic to fully quantum that could be adopted in different situations. They reported that this approach could account for many of the phenomena of human decision making that are not accounted for by the classic model alone. Furthermore, they provided evidence that the transition from quantum representation to a more classic representation happens as the subject gains more familiarity with the task. ${ }^{38}$

\section{Randomness and free will}

One the most consequential aspects of psychiatry is its relation to matters of responsibility, freedom of decision, capacity of judgment and autonomy. Psychiatrists are very often called upon to give expert opinions in relation to important questions regarding the mental state of their patients, such as their capacity to make decisions regarding crucial aspects of their lives (e.g., accept or refuse treatment, criminal responsibility). These questions, which have important consequences for patients and society, invoke to a certain degree the question of free will. For example, when a patient is considered noncriminally responsible because of mental illness, this implies that the patient committed a criminal action while deprived of their free will and thus does not bear responsibility. It is therefore important for researchers and clinicians in the field of psychiatry to confront the question of free will - one of the oldest and most debated questions in the history of philosophy.

While any detailed discussion of this question is beyond the scope of this editorial, it is noteworthy that all forms of determinism (e.g., scientific, religious, logical) as well as randomness have been considered fundamental threats to free will. Indeed, determinism in its modern version (causal determinism) schematically postulates that, given the laws of nature and what happened in our past, all our present and future decisions/actions are predetermined and agents do not have the option to act otherwise. On the other hand, if randomness is behind our decisions/actions, this means that we are not the true generators of our ac- tions and that they happen randomly, which seems to be an even more serious threat to free will.

While the argument of the incompatibility of free will with determinism is relatively easy to grasp given our familiarity with the concepts of causality and determinism, the relation of free will to indeterminism is a much trickier question given that our grasp of indeterminism and its relation to randomness is much less intuitive and has been developed only recently compared with the centuries-old concepts of determinism and causality. In a paper titled, "Free will is compatible with randomness," Calude and colleagues point to the fact that randomness is a complex construct that comes in different degrees (e.g., dice tossing, quantum physics and Martin-Löf randomness are all mathematically proven different forms of randomness) and that, mathematically, the so-called "pure randomness" and "true randomness" are vacuous concepts. ${ }^{39}$ They also show that randomness and indeterminism do not imply one another and conclude that, after developing several scenarios of decision making, free will is compatible with randomness and that indeterminism is a necessary condition for free will. The tenets of this view of free will, also called libertarianism, hold that fundamental aspects of human values (e.g., moral responsibility, worth blame) are not possible if our actions are causally determined by the laws of nature and our history. Robert Kane is a representative of the libertarian school who made significant contributions to the comprehensibility of free will in the last 20 years. One of his main contributions is the introduction of the notion of self-forming action: action (generally morally laden) that is decided and executed under particular circumstances (e.g., time pressure, high stress, ambiguity, unfamiliar situations). Of note, it is under these conditions that decision making and actions might operate at the edges of chaos, with clear possibility of randomness and nondeterministic processes playing a role, as suggested by the experimental work of Trueblood and colleagues. ${ }^{38}$ These decisions/actions punctuate our lives and ultimately make us who we are. It is through these decisions/ actions that we are held accountable, praiseworthy, or blameworthy and responsible. ${ }^{40}$ In a book titled Four views on free will, the authors write, "Indeterminism paradoxically opens up the genuine possibility of pursuing other purposes - of choosing or doing otherwise - in accordance with, rather than against, our wills (voluntarily) and reasons (rationally). To be self-forming agents (creators of ourselves) - to have free will - there must at times in life be obstacles and hindrances in our wills of this sort that we must overcome." They add, "Each undetermined self-forming free choice is a value experiment whose justification lies in the future and is not fully explained by past reasons. In making such a choice, we say, in effect, 'let's try this.' It is not required by my past, but it is consistent with my past and is one branching pathway in the garden of forking paths my life can now meaningfully take." 41 These recent developments in the cognitive and philosophical concepts of free will are now ripe for a neurocognitive formulation of this hard question. ${ }^{42}$ 
Thus, if indeterminism opens up the capacity of doing otherwise and engaging in free actions, we propose that mental illnesses, where the capacity to do otherwise is often curtailed (e.g., under the effect of delusion, substance dependence, obsession, depressive cognitive distortions), may be associated with overdeterminism that may originate from biological and/or psychological processes. In this sense, some mental disorders may be conceived as pathologies of freedom.

\section{Conclusion}

We began this editorial by introducing the fundamental notions of randomness and selection, as conceived in the field of evolution, and then proceeded to illustrate their pervasiveness at all phases and junctures of life. Here we conclude by giving alternative names to these processes names that are more in line with this extended framework: generative and inventive processes, respectively. The generative process corresponds to random generation of different possibilities via multiple mechanisms, opening the door to Borges' Garden of Forking Paths and the inventive process that leads to selecting one possibility via different mechanisms (environmental pressure, quantum collapse or libertarian free will). A dynamic interplay between these generative and inventive processes can lead to singular trajectories that we, as clinicians and scientists, recognize and label as mental illnesses.

Family and twin studies guided generations of psychiatric clinicians and researchers in the idea that genetic determinism contributes significantly to most major mental disorders. This scientific knowledge might appear to contradict some cultural beliefs. For example, a North African proverb asserts that "madness, we got stuck in it; sound minds are out of our seeds (genes)." However, after a long clinical and research experience in psychiatry, this ancestral wisdom appears to us much less contradictory to our scientific knowledge. Indeed, when we say, for example, that $80 \%$ of the variance in phenotype schizophrenia is attributable to additive genetic factors, this statement is usually construed in a very reductionist view because of the ambiguity of what we consider to be schizophrenia. We argue that a phenotype like schizophrenia is consubstantial with other major phenotypes, such as intelligence, emotions and personality traits, and it cannot exist in a void. Under this conception, it is not surprising that hundreds if not thousands of genetic variations, dispersed all over the genome are implicated in schizophrenia (and most of other mental disorders) and that heritability of these disorders is often very high. This high heritability captures not only the symptoms of mental disorders (per DSM criteria), but also all the other consubstantial phenotypes that are essential to the expression of whatever symptoms we call mental illnesses. The fact that genome-wide association studies have shown that major mental disorders, cognitive abilities and personality traits show important genetic correlations is consistent with this view. Consequently, what role genetics play in what is most intuitively (and saliently) considered madness (e.g., hallucinations, delusions) may be trivial, and these symptoms might arise from the vagaries of randomness at all the levels discussed here.

Some implications may be derived from these views for psychiatry. First, in medicine and particularly in psychiatry, we cherish the notion of individuality. The framework presented here can help us to better understand this individuality at different levels of analyses, to share this understanding with our patients and their families, and to inject humility in our clinical and research practices. Second, there is probably a limit to what we can learn from biological psychiatry, conceived and practised within a deterministic framework. The nature and amount of randomness that is fossilized in each individual is likely too high to be solved through deterministic approaches. One important question that follows is whether we are now operating in the margins of this limit, where nondeterministic variance drastically limits our capacity to identify deterministic factors that account for a clinically meaningful portion of variance in psychiatric phenotypes. The dearth of clinically meaningful discoveries from biological psychiatry in the last few decades makes this question pertinent and legitimate. Some will argue that only time can answer this question. But how long? Finally, while nondeterministic (along with deterministic) biological processes can engage the developmental trajectories of individuals on the path of mental illnesses, psychological nondeterministic processes may be our path to free will and, thus, our capacity to change our life trajectories and to recover from mental illnesses. As health professionals, we are always meeting our patients in times of uncertainty, stress and ambiguity. This is when we need to cultivate the seeds of positive change.

Acknowledgements: The authors thank Drs. Patricia Boksa and Ashok Malla for their comments and suggestions on this editorial.

Affiliations: From the Department of Psychiatry, McGill University, Montreal, Que., Canada (Joober, Karama); and the Douglas Hospital Research Centre, Montreal, Que., Canada (Joober, Karama).

Competing interests: See jpn.ca for R. Joober. None declared for S. Karama.

Content licence: This is an Open Access article distributed in accordance with the terms of the Creative Commons Attribution (CC BY-NC-ND 4.0) licence, which permits use, distribution and reproduction in any medium, provided that the original publication is properly cited, the use is noncommercial (i.e., research or educational use), and no modifications or adaptations are made. See: https: / / creativecommons.org/licenses /by-nc-nd/4.0/

\section{References}

1. Nachman MW, Crowell SL. Estimate of the mutation rate per nucleotide in humans. Genetics 2000;156:297-304.

2. Otto SP, Lenormand T. Resolving the paradox of sex and recombination. Nat Rev Genet 2002;3:252-61.

3. Joober R, Tabbane K. From the neo-Kraepelinian framework to the new mechanical philosophy of psychiatry: regaining common sense. J Psychiatry Neurosci 2019;44:3-7.

4. Eagle A. Chance versus randomness. In: Edward N. Zalta, editor. The Stanford Encyclopedia of Philosophy; 2021. Available: https:/ / plato.stanford.edu/archives/spr2021/entries/chance -randomness/ (accessed 2021 Aug. 6).

5. Hoefer C. Causal determinism. In: Edward N. Zalta, editor. The Stanford Encyclopedia of Philosophy; 2016. Available: https:// 
plato.stanford.edu/archives/spr2016/entries/determinism -causal (accessed 2021 Aug. 6).

6. Bressloff PC. Stochastic processes in cell biology. Springer International Publishing; 2021.

7. Symmons O, Raj A. What's luck got to do with it: single cells, multiple fates, and biological nondeterminism. Mol Cell 2016;62:788-802.

8. Elowitz MB, Levine AJ, Siggia ED, et al. Stochastic gene expression in a single cell. Science 2002;297:1183-6.

9. Ozbudak EM, Thattai M, Kurtser I, et al. Regulation of noise in the expression of a single gene. Nat Genet 2002;31:69-73.

10. Golding I, Paulsson J, Zawilski SM, et al. Real-time kinetics of gene activity in individual bacteria. Cell 2005;123:1025-36.

11. Swain PS, Elowitz MB, Siggia ED. Intrinsic and extrinsic contributions to stochasticity in gene expression. Proc Natl Acad Sci U S A 2002;99:12795-800.

12. Khare A, Shaulsky G. First among equals: competition between genetically identical cells. Nat Rev Genet 2006;7:577-83.

13. Levayer R, Hauert B, Moreno E. Cell mixing induced by myc is required for competitive tissue invasion and destruction. Nature 2015;524:476-80.

14. Guillemin A, Duchesne R, Crauste F, et al. Drugs modulating stochastic gene expression affect the erythroid differentiation process. PLoS One 2019;14:e225166.

15. Losick R, Desplan C. Stochasticity and cell fate. Science 2008;320:65-8.

16. Moris N, Pina C, Arias AM. Transition states and cell fate decisions in epigenetic landscapes. Nat Rev Genet 2016;17:693-703.

17. Czyz W, Morahan JM, Ebers GC, et al. Genetic, environmental and stochastic factors in monozygotic twin discordance with a focus on epigenetic differences. BMC Med 2012;10:93.

18. Ballouz S, Pena MT, Knight FM, et al. The transcriptional legacy of developmental stochasticity. BioRxiv; 2019. doi: https://doi. org /10.1101/2019.12.11.873265.

19. Skuse DH, James RS, Bishop DV, et al. Evidence from Turner's syndrome of an imprinted X-linked locus affecting cognitive function. Nature 1997;387:705-8.

20. Girard SL, Dion PA, Bourassa CV, et al. Mutation burden of rare variants in schizophrenia candidate genes. PLoS One 2015; 10:e0128988.

21. Igolkina AA, Armoskus C, Newman JRB, et al. Analysis of gene expression variance in schizophrenia using structural equation modeling. Front Mol Neurosci 2018;11:192.

22. Kebir O, Chaumette B, Krebs MO. Epigenetic variability in conversion to psychosis: novel findings from an innovative longitudinal methylomic analysis. Transl Psychiatry 2018;8:93.

23. van der Meulen M, Wierenga LM, Achterberg M, et al. Genetic and environmental influences on structure of the social brain in childhood. Dev Cogn Neurosci 2020;44:100782.
24. Linneweber GA, Andriatsilavo M, Dutta SB, et al. A neurodevelopmental origin of behavioral individuality in the Drosophila visual system. Science 2020;367:1112-9.

25. Yao Y, Palaniyappan L, Liddle P, et al. Variability of structurally constrained and unconstrained functional connectivity in schizophrenia. Hum Brain Mapp 2015;36:4529-38.

26. Deco G, Rolls ET, Romo R. Stochastic dynamics as a principle of brain function. Prog Neurobiol 2009;88:1-16.

27. Rolls ET, Deco T. The Noisy Brain: Stochastic Dynamics as a Principle of Brain Functioning. Oxford University Press; 2012

28. Deco G, Rolls ET, Albantakis L, et al. Brain mechanisms for perceptual and reward-related decision-making. Prog Neurobiol 2013;103:194-213.

29. Rolls ET, Deco G. A computational neuroscience approach to schizophrenia and its onset. Neurosci Biobehav Rev 2011;35: 1644-53.

30. Tversky A, Kahneman D. Judgment under uncertainty: heuristics and biases. Science 1974;185:1124-31.

31. Tversky A, Kahneman D. Extensional versus intuitive reasoning: the conjunction fallacy in probability judgment. Psychol Rev 1983;90:293-315.

32. Pothos EM, Busemeyer JR. Quantum principles in psychology: the debate, the evidence, and the future. Behav Brain Sci 2013;36:310-27.

33. Pothos EM, Busemeyer JR. Can quantum probability provide a new direction for cognitive modeling? Behav Brain Sci 2013;36: 255-74.

34. Pothos EM, Busemeyer JR. Formalizing heuristics in decisionmaking: a quantum probability perspective. Front Psychol 2011;2:289.

35. Pothos EM, Busemeyer JR. A quantum probability explanation for violations of 'rational' decision theory. Proc Biol Sci 2009;276: 2171-8.

36. Busemeyer JR, Wang Z, Pothos E. Quantum Models of Cognition and Decision. Oxford Handbooks Online; 2015.

37. Kvam PD, Busemeyer JR, Pleskac TJ. Temporal oscillations in preference strength provide evidence for an open system model of constructed preference. Sci Rep 2021;11:8169.

38. Trueblood JS, Yearsley JM, Pothos EM. A quantum probability framework for human probabilistic inference. J Exp Psychol Gen 2017;146:1307-41.

39. Calude CSKF, Poznanovic N. Free will is compatible with randomness. Philosophical Inquiries 2016;4:37-52.

40. Kane R. The Significance of Free Will. Oxford Scholarship Online; 2003.

41. Fisher JM, Kane R, Pereboom D, et al. Four views on free will. Australia: Blackwell Publishing; 2007.

42. Hills TT. Neurocognitive free will. Proc Biol Sci 2019;286: 20190510. 